The University of San Francisco

USF Scholarship: a digital repository @ Gleeson Library |

Geschke Center

2004

\title{
Nucleosynthesis, Reionization, and the Mass Function of the First Stars
}

Jason Tumlinson

Aparna Venkatesan

University of San Francisco, avenkatesan@usfca.edu

J Shull

Follow this and additional works at: http://repository.usfca.edu/phys

Part of the Astrophysics and Astronomy Commons, and the Physics Commons

\section{Recommended Citation}

Tumlinson, J., Venkatesan, A., \& Shull, J. (2004). Nucleosynthesis, reionization, and the mass function of the first stars. Astrophysical Journal, 612(2), 602-614. doi:10.1086/422571

This Article is brought to you for free and open access by the College of Arts and Sciences at USF Scholarship: a digital repository @ Gleeson Library | Geschke Center. It has been accepted for inclusion in Physics and Astronomy by an authorized administrator of USF Scholarship: a digital repository @ Gleeson Library | Geschke Center. For more information, please contact repository@usfca.edu. 
The Astrophysical Journal, 612:602-614, 2004 September 10

(C) 2004. The American Astronomical Society. All rights reserved. Printed in U.S.A.

\author{
NUCLEOSYNTHESIS, REIONIZATION, AND THE MASS FUNCTION OF THE FIRST STARS \\ Jason Tumlinson, ${ }^{1}$ Aparna Venkatesan, ${ }^{2,3}$ and J. Michael Shull ${ }^{2,4}$ \\ Received 2004 January 18; accepted 2004 May 13
}

\begin{abstract}
We critique the hypothesis that the first stars were very massive stars (VMSs; $M>140 M_{\odot}$ ). We review the two major lines of evidence for the existence of VMSs: (1) that the relative metal abundances of extremely metalpoor Galactic halo stars show evidence of VMS enrichment and (2) that the high electron-scattering optical depth $\left(\tau_{e}\right)$ to the cosmic microwave background found by the Wilkinson Microwave Anisotropy Probe (WMAP) requires VMSs for reionization in a concordance $\Lambda$ CDM cosmology. The yield patterns of VMSs exploding as pairinstability supernovae are incompatible with the Fe-peak and $r$-process abundances in halo stars. Models including Type II supernovae and/or "hypernovae" from zero-metallicity progenitors with $M=8-40 M_{\odot}$ can better explain the observed trends. We use the nucleosynthesis results and stellar evolution models to construct an initial mass function (IMF) for reionization. With a simple metal transport model, we estimate that halo enrichment curtails metal-free star formation after $\sim 10^{8}$ yr at $z \sim 20$. Because the lifetime-integrated ionizing photon efficiency of metal-free stars peaks at $\sim 120 M_{\odot}$ and declines at higher mass, an IMF with an approximate lower bound at $M \sim 10-20 M_{\odot}$ and no VMS can maximize the ionizing photon budget and still be consistent with the nucleosynthetic evidence. An IMF devoid of low-mass stars is justified independently by models of the formation of primordial stars. Using a semianalytic model for $\mathrm{H}$ I and $\mathrm{He}$ II reionization, we find that such an IMF can reproduce $\tau_{e} \simeq 0.10-0.14$, consistent with the range from $W M A P$, without extreme astrophysical assumptions, provided that metal-free star formation persists $10^{7}-10^{8} \mathrm{yr}$ after star formation begins. Because stars in the mass range $50-140 M_{\odot}$ are the most efficient sources of ionizing photons but are expected to collapse to black holes without releasing metals, this IMF effectively decouples early metal enrichment and early ionization. Such an IMF may allow the unique properties of the zero-metallicity IMF to persist longer than they would in the pure VMS case and to contribute significantly to the global ionizing photon budget before halo self-enrichment and/or interhalo metal transport truncates metal-free star formation. We conclude, on the basis of these results, that VMSs are not necessary to meet the existing constraints commonly taken to motivate them.
\end{abstract}

Subject headings: cosmology: theory — nuclear reactions, nucleosynthesis, abundances stars: luminosity function, mass function

\section{INTRODUCTION}

Theoretical ideas about the first, metal-free stars have developed rapidly in recent years, despite the absence of direct observational evidence of their properties. As a specific prediction of the big bang theory, metal-free first stars confront both cosmology and astrophysics generally with important questions: When did the first stars form? What was their initial mass function (IMF)? What were their lifetimes and feedback effects? Where are their remnants (compact objects and/or metal enrichment)? The standard tools of stellar evolution theory have been applied to calculate the evolving structure and radiative spectra of metal-free stars, given their mass (Tumlinson \& Shull 2000; Marigo et al. 2001; Bromm et al. 2001; Schaerer 2002; Tumlinson et al. 2003, hereafter TSV03). Many uncertainties remain about the cosmological context and feedback effects of the first stars, the largest of which concern the duration of the metal-free phase and the IMF. Despite these

\footnotetext{
1 Department of Astronomy and Astrophysics, University of Chicago, 5640 South Ellis Avenue, Chicago, IL 60637.

2 Center for Astrophysics and Space Astronomy, UCB 389, Department of Astrophysical and Planetary Sciences, University of Colorado, Boulder, CO 80309-0389.

${ }^{3}$ National Science Foundation Astronomy and Astrophysics Postdoctoral Fellow.

${ }^{4}$ Also at JILA, University of Colorado, and National Institute of Standards and Technology.
}

unknowns, theoretical progress on the first stars has arrived at six points of consensus:

1. The first stars form from metal-free gas in the first collapsed dark matter halos around $z \sim 20$ (Ricotti et al. 2002a, 2002b).

2. The first stars may be very massive $\left(M>100 M_{\odot}\right)$ because they are restricted during their formation to inefficient cooling by molecular hydrogen below $10^{4} \mathrm{~K}$ (Abel et al. 2002).

3. These very massive first stars seed their own halos and possibly enrich nearby ones by releasing metals from pairinstability supernovae (140-260 $M_{\odot}$; Heger \& Woosley 2002, hereafter HW02).

4. At critical metallicity $Z \gtrsim 10^{-3.5} Z_{\odot}$, protostellar clouds are able to cool and fragment more efficiently, leading to a "normal" IMF (Bromm \& Loeb 2003; Schneider et al. 2002). Where this metallicity has not been reached, the IMF retains its unusual properties. Halo and intergalactic medium (IGM) enrichment probably occur inhomogeneously in time and space during the transition to "normal" star formation.

5. The first stars begin and may complete the reionization of intergalactic H I (Gnedin \& Ostriker 1997) and He II (Venkatesan et al. 2003, hereafter VTS03). The total first-stars contribution to the global ionizing photon budget is unknown, but the efficient ionization of very massive stars (VMSs) is thought to be required. There may be partial recombination during the transition phase in metallicity, followed by a second reionization near $z_{r}=6$ (Cen 2003a; Wyithe \& Loeb 2003; VTS03). 
6. The first-stars epoch ends when all star-forming regions have achieved the critical metallicity. This is thought to occur before the final stages of $\mathrm{H}$ I reionization at $z_{r}=6.2$.

This consensus is not universally accepted in all details, but it serves as a working hypothesis in the literature. Point 2, the "VMS hypothesis," has appeared often in studies of the first stars concerned with their cosmic origins, their sites of formation, and their importance for reionization. In this paper, we examine this hypothesis with the objective of constructing an IMF for the first stars that is consistent with current observational constraints. We meet this goal by examining the arguments for the VMS hypothesis and by investigating how its key lines of evidence can also support an IMF without VMSs.

Our critique of the VMS hypothesis addresses consensus points 3 and 5 particularly. The empirical basis of point 3 is the work of Oh et al. (2001) and Qian \& Wasserburg (2002), who argued that the relative metal abundances in extremely metal-poor (EMP) halo stars match the expected signatures from VMSs (HW02). Their conclusion has since been adopted as positive evidence of VMS metal enrichment and ionizing photon production in the first stellar generation. We argue that this line of evidence is not supported by the broader sets of abundance data presently available. Instead, we adopt the view of Umeda \& Nomoto (2003b, hereafter UN03b) that these observed abundances are better matched by core-collapse supernovae or "hypernovae" from 10 to $50 M_{\odot}$ progenitors. We then examine point 5 in light of evolving spectra models for metal-free stars (TSV03) and recent studies of star formation at zero metallicity. VMSs have been suggested as the most viable cause of the high Thomson electron-scattering optical depth $\tau_{e}=0.17_{-0.07}^{+0.08}$ derived from the cosmic microwave background (CMB) data obtained by the Wilkinson Microwave Anisotropy Probe (WMAP; Spergel et al. 2003). Instead, we find that reionization models incorporating an IMF that excludes both low-mass stars and VMSs can meet the reionization constraint. The lower but equally uncertain value $\tau_{e}=0.12_{-0.06}^{+0.08}$ found by joint analysis of $W M A P$ and the Sloan Digital Sky Survey (SDSS) matter power spectrum by Tegmark et al. (2004) is reproduced by our models, but since it is also easier to accommodate to a standard IMF, we conclude that the optical depth to reionization is not a decisive constraint on the IMF at early times. Because the ionizing efficiency of metal-free stars increases more rapidly from 1 to $50 M_{\odot}$ than it does above this range (see $\S 4$ ), we are able to construct an IMF with ionizing properties similar to those of VMSs. We conclude, on the basis of these results, that VMSs are not necessary to meet the existing constraints commonly taken to motivate them. We also do not need to invoke high values for the escape fraction of ionizing radiation or the star formation efficiency in halos (cf. Oh et al. 2001; Somerville et al. 2003).

This paper is structured as follows. First, in $\S 2$, we review the theoretical motivation for VMSs in the first stellar generation and distinguish two versions of the VMS hypothesis. In $\S 3$ we review the extremely metal-poor halo star abundances and compare specific patterns in the nucleosynthetic evidence with theoretical yields. In $\S 4$ we use our results to motivate a range of possible first-stars IMFs and derive a timescale for the epoch of metal-free star formation. In $\S 5$ we discuss semianalytic reionization models that compare these different IMFs with VMSs. In $\S 6$ we draw general conclusions and discuss some unsolved problems in the study of the first stars.

\section{THE VMS HYPOTHESIS}

We define VMS to include stars with $M \geq 140 M_{\odot}$, the lowest mass at which metal-free stars explode as pairinstability supernovae (PISNe; HW02). This is the only clear mass cutoff higher than the uncertain $M \sim 40-70 M_{\odot}$ division between core-collapse supernovae ( $\mathrm{SNe}$ ) and complete collapse to black holes (HW02). The $M \geq 140 M_{\odot}$ definition cleanly separates two mass regimes with very different nucleosynthetic signatures (see $\S 3$ ) but similar radiative properties. The mass cutoff is rigid for the purposes of definition only; it may be more loosely defined in reality without interfering with our conclusions, provided that it marks a qualitative change in the nucleosynthetic signature of the stars.

The theoretical motivation for the VMS hypothesis rests primarily on the microscopic processes of radiative cooling in low-density primordial gas. At $T \lesssim 10^{4} \mathrm{~K}$, cooling by $\mathrm{H}$ and $\mathrm{He}$ is negligible and metal line cooling is dominated by [C II], [O I], and [Si II] fine-structure lines. Because metal-free gas is restricted to relatively inefficient cooling by $\mathrm{H}_{2}$ at $T \lesssim 10^{4} \mathrm{~K}$, cooling rates are reduced, and the balance between pressure support and gravitational collapse is shifted to higher temperatures. Primordial protostellar objects, being unable to cool or fragment, must therefore be more massive to overcome their elevated gas pressure. The final star should also be more massive if the entire cloud can accrete. This simple physical description was rigorously implemented by Abel et al. (2002), who used an adaptive mesh refinement hydrodynamics code to follow the evolution of metal-free gas from cosmological initial conditions down to the formation of a self-gravitating object with $M \sim 600 M_{\odot}$. The simulation was halted when the core became optically thick to $\mathrm{H}_{2}$ line emission, which occurred before the object was completely formed. This study implies VMSs with $M>140 M_{\odot}$ if all the gravitationally bound gas can accrete to a single star.

Recent studies (Omukai \& Palla 2003; Tan \& McKee 2002; Bromm \& Loeb 2004), taking the Abel et al. (2002) result as a starting point for semianalytic models of primordial star formation, indicate that reality is complicated by stellar feedback on the accreting matter. Omukai \& Palla (2003) identified a critical accretion rate $\left(\dot{M}=4 \times 10^{-3} M_{\odot} \mathrm{yr}^{-1}\right)$ below which laminar accretion onto a spherically symmetric protostar can proceed to $M>100 M_{\odot}$ unimpeded by feedback. Above this critical rate, the accretion luminosity exceeds the Eddington limit and the flow is reversed at 100-300 $M_{\odot}$. Models by Bromm \& Loeb (2004) have found a conservative upper limit of $500 M_{\odot}$ by setting the time available to accretion to be the stellar lifetime $\left(\sim 3 \times 10^{6} \mathrm{yr}\right)$ but ignoring feedback. Tan \& McKee (2002) consider the effects of disk accretion geometry, rotation, and radiation feedback in limiting the mass of primordial stars. They find that these feedback mechanisms are likely to operate at $M=30-100 M_{\odot}$, perhaps limiting the masses of metal-free stars to this range. However, they later found (Tan \& McKee 2004) that none of the feedback mechanisms considered can halt the accretion before the star achieves $\sim 30 M_{\odot}$. This important result indicates that both VMSs and low-mass stars may not form at zero metallicity and suggests that the mass limits of the primordial IMF may be quite different from today. In $\S 4$ we use this idea and empirical constraints to construct an IMF for the first stars.

These theoretical studies of primordial protogalaxies and star formation posit that the first generation of stars was partially, and perhaps completely, composed of VMSs. To capture existing evidential claims, we introduce a distinction between 
the strong VMS hypothesis ("the first generation were exclusively VMSs") and the weak VMS hypothesis ("the first generation included VMSs in addition to $M \lesssim 50 M_{\odot}$ stars"). Both versions appear in the literature although not with these names. The evidence can bear differently on the two versions of the hypothesis because the ionizing properties and nucleosynthetic yields vary across the mass function. These labels do not connote the relative merit of the two versions; they suggest only that the "strong" hypothesis is more readily falsified than the "weak." The weak hypothesis is expected to be more difficult to constrain because it includes low-mass stars and so is more like the present-day circumstances. We evaluate these versions of the hypothesis below.

The HW02 stellar evolution and supernova models show that between 140 and $260 M_{\odot}$, zero-metallicity stars are highly susceptible to the pair-production instability after core $\mathrm{He}$ depletion. This instability quickly disrupts the star, ejects metals, and leaves no compact remnant. Because it is triggered at an unusually early period in the star's evolution, this PISN produces an unusual nucleosynthetic signature. If the VMS hypothesis is correct, the signature of PISNe could appear in the metal abundances of "second generation" EMPs in the Galactic halo (see $\S 3$ ). Qian \& Wasserburg (2002) and $\mathrm{Oh}$ et al. (2001) used this idea to argue that these stars justify the VMS hypothesis.

Models with VMSs have also been proposed to explain the current data on IGM reionization, specifically the high electron-scattering optical depth $\tau_{e}$ found in the WMAP CMB data. For the simplest possible reionization history (a step function at fixed $z_{r}$ ), this result implies that complete reionization occurred at $z_{r}=17 \pm 4$ (Spergel et al. 2003; Kogut et al. 2003). Recent work that combines the three-dimensional matter power spectrum, $P(k)$, from the SDSS with $W M A P$ data finds that $\tau_{e}=0.12_{-0.06}^{+0.08}$ (Tegmark et al. 2004). The large uncertainties arise from the fact that the first-year $W M A P \tau_{e}$ results were based on temperature-polarization correlations, which are an indirect measure of $\tau_{e}$. Additional WMAP data will allow autocorrelation of the polarized CMB maps and should provide a direct and more precise determination of $\tau_{e}$.

When joined with spectroscopic observations that indicate a rapidly rising $\mathrm{H}_{\mathrm{I}}$ Gunn-Peterson opacity up to $z \sim 6.4$ (Becker et al. 2001; Djorgovski et al. 2001), the WMAP result implies that the IGM experienced a relatively complex ionization history, with extended periods of ionization in $\mathrm{H} \mathrm{I}$ and/or He II at $z \sim 6-20$ (VTS03; Cen 2003a; Wyithe \& Loeb 2003). In a study of the ionizing properties of VMSs, Bromm et al. (2001) found VMSs with $M=100-1000 M_{\odot}$ to be an order of magnitude more efficient, on the zero-age main sequence, at generating ionizing photons per unit stellar mass than are stars in a normal IMF with $M=1-100 M_{\odot}$ (Tumlinson \& Shull 2000). VMSs produce efficient ionization and have short lifetimes, and they are thought to form in the metal-free gas of collapsed halos at high $z$. In light of these features, scenarios involving VMSs in combination with subsequent stellar populations have been widely proposed as an attractive explanation for the observed ionization and thermal history of the IGM (Hui \& Haiman 2003) and the large detected $\tau_{e}$ to the CMB (Cen 2003b; Haiman \& Holder 2003; Fukugita \& Kawasaki 2003; Ciardi et al. 2003; Kaplinghat et al. 2003).

These "nucleosynthesis" and "reionization" lines of evidence are often taken to support the VMS hypothesis. But are VMSs required? Are there other viable models for the EMP metal abundances and reionization that do not include VMSs?
We argue that neither line of evidence is compelling. We take up the nucleosynthesis arguments first.

\section{THE FIRST STARS AND NUCLEOSYNTHESIS}

\subsection{General Patterns in the Nucleosynthetic Evidence}

Many studies of reionization by the first stars ( $\S 2$ ) have adopted the VMS hypothesis, citing as evidence the apparent match between PISN yields and the metal abundances of EMP halo stars (Oh et al. 2001; Qian \& Wasserburg 2002). A full review of the literature on metal abundances (elements $\mathrm{Li}-\mathrm{U}$ ) in EMP stars is beyond the scope of this study. The review by McWilliam (1997) gives a survey up to that time, and papers that cite it (available from the Astrophysical Data Service) provide useful entry points to the more recent literature. We focus here on three clear features in Galactic halo EMP abundances that are confirmed by multiple studies and that bear directly on the VMS hypothesis. These are the specific Fe-peak element ratios (especially $\mathrm{Zn}$ ), the widespread presence of $r$-process elements, and elevated $[\mathrm{C} / \mathrm{Fe}, \mathrm{N} / \mathrm{Fe}$, $\mathrm{O} / \mathrm{Fe}]$ ratios. We summarize these features here, and in $\S 3.2$ we compare them in detail with theoretical models incorporating the VMS hypothesis.

Iron-peak elements $(\mathrm{Cr}-\mathrm{Zn})$.-Because these elements are produced in explosive events, they are sensitive to the energy, rotation, mass cut, and asymmetry of the supernova and to the pre-SN stellar properties. Large samples presented by McWilliam et al. (1995) and Carretta et al. (2002) found that the Fe-peak ratios to Fe qualitatively change their behavior at $[\mathrm{Fe} / \mathrm{H}] \simeq-3$. Below this metallicity, $[\mathrm{Zn} / \mathrm{Fe}]$ and $[\mathrm{Co} / \mathrm{Fe}]$ increase while $[\mathrm{Cr} / \mathrm{Fe}]$ and $[\mathrm{Mn} / \mathrm{Fe}]$ decrease. This trend was interpreted as a change in the nature of the supernova progenitors, and the increased scatter was attributed to inhomogeneous enrichment. The more recent and higher resolution study by Cayrel et al. (2004) found lower scatter and a smooth rather than abrupt change in the abundance ratios at $[\mathrm{Fe} / \mathrm{H}] \simeq$ -3 to -4 . In any case, the overall abundance pattern is roughly consistent across the three studies, and it provides a good test for theoretical yields.

$r$-process elements $(A>100)$.- These elements are thought to be produced by rapid neutron capture in hot, dense, neutron-rich environments during explosive events. Although the exact physical sites are still uncertain, the proposed mechanisms are all associated with massive stars in the range $M=8-40 M_{\odot}$ (Truran et al. 2002). The absolute abundances and relative ratios of $r$-process elements are thus sensitive indicators of core-collapse supernova activity. The existing samples of EMP stars show widespread evidence of $r$-process elements down to $[\mathrm{Fe} / \mathrm{H}] \sim-3$ (McWilliam et al. 1995; Burris et al. 2000). The mean $[r / \mathrm{Fe}]$ is similar to the solar value at all $[\mathrm{Fe} / \mathrm{H}]$ but with up to 2 dex scatter at $[\mathrm{Fe} / \mathrm{H}] \sim$ -3 . The relative abundances (i.e., $[\mathrm{Eu} / \mathrm{Ba}]$ ) are also similar to the solar values.

Primary elements (C, N, O).- - Of these direct products of main-sequence stellar nucleosynthesis, $\mathrm{C}$ is easily found in EMPs, but $\mathrm{N}$ and $\mathrm{O}$ are difficult to measure. Many EMP stars are C-rich relative to Fe. Further study of this class of stars may ultimately reveal them to be iron-poor rather than metalpoor and therefore less chemically primitive than their low $[\mathrm{Fe} / \mathrm{H}]$ makes them appear. As pointed out by Bromm \& Loeb (2003), C and not Fe is the dominant interstellar coolant that may influence the early IMF. As shown in TSV03, $\mathrm{C}$ is the nuclear burning catalyst that determines by its absence the unusual behavior of the first stars. These factors favor $\mathrm{C}$ 
instead of $\mathrm{Fe}$ as the "reference element" for finding chemically primitive stars in the local universe. By this measure, these $\mathrm{C}$-enhanced stars may be less primitive than those with higher $[\mathrm{Fe} / \mathrm{H}]$. For now, however, we follow the observational studies by assuming that they are in same class as the other EMPs.

\subsection{VMSs and Nucleosynthesis}

In this section, we describe the nucleosynthetic evidence for VMSs as stated in the literature. Observational studies of EMP metal abundances have regarded the trends in the Fe-peak and $r$-process elements as the most critical constraints on the nature of the first stars. We examine the model of Wasserburg \& Qian (2000) and Qian \& Wasserburg (2002), which represents only one example from a broad class of possible models that could explain the observed variations. We cannot address all the possible variations in such a class, so we use this one because it admits VMS objects as a possible source of early enrichment. We later use the phenomenological model of Fields et al. (2002) to illustrate why a successful model for the observed trends in $r$-process abundances has difficulty incorporating VMSs.

Wasserburg \& Qian (2000; hereafter WQ00) examined the behavior of $[r / \mathrm{Fe}]$ with $[\mathrm{Fe} / \mathrm{H}]$ in the McWilliam et al. (1995) sample and argued that the qualitative change in $[r / \mathrm{Fe}]$ at $[\mathrm{Fe} / \mathrm{H}] \lesssim-3$ presented an "iron conundrum," namely, that the wide dispersion in $\mathrm{Eu}$ and $\mathrm{Ba}$ abundances at $[\mathrm{Fe} / \mathrm{H}] \simeq-3$ suggested unrelated sources of Fe and $r$-elements. In response, they proposed a "prompt" (P) inventory of Fe production by an initial population with large Fe yields but little or no $r$ production. This initial inventory ceased at $[\mathrm{Fe} / \mathrm{H}] \sim-3$, where the onset of high-frequency (HF) events ( $\mathrm{SNe}$ II; $\tau \sim 10^{7} \mathrm{yr}$ ) and low-frequency (LF) events (SNe Ia; $\tau \sim 10^{8}$ ) initiated a correlation between $r$ and $\mathrm{Fe}^{5}$ In this model, the wide variation in $\mathrm{Fe}$ for small barium abundance $\epsilon(\mathrm{Ba})$ is attributed to diverse $\mathrm{Fe}$ yields for $\mathrm{P}$ events or to multiple $\mathrm{P}$ progenitors. The low $\epsilon(\mathrm{Ba})$ at $[\mathrm{Fe} / \mathrm{H}] \lesssim-3$ is attributed to small coproduction of $r$-elements by $\mathrm{P}$ events or to dilution of a modest HF contribution. WQ00 made no specific claims about the mass or metallicity of the P inventory sources.

After establishing the general properties of the $\mathrm{P}$ inventory in WQ00, Qian \& Wasserburg $(2001,2002)$ noted that VMSs of a few hundred $M_{\odot}$ possess the Fe-rich, $r$-poor yields and short lifetimes (1-3 Myr) necessary to produce the $\mathrm{P}$ inventory Fe over the short time $\left(\sim 10^{7} \mathrm{yr}\right)$ before the onset of HF events. This VMS model readily accommodates the qualitative change in observed abundances at $[\mathrm{Fe} / \mathrm{H}]=-3$, near the $Z=10^{-3.5} Z_{\odot}$ threshold where metal line cooling is expected to change the IMF (Bromm \& Loeb 2003). This model was extended by Oh et al. (2001) to link the VMS hypothesis to reionization. They pointed out that the trends in $[\mathrm{Fe} / \mathrm{H}]$ and $\mathrm{Ba}$ also appear in $\mathrm{Si}$ and $\mathrm{Ca}$, elements produced by PISNe, but not in $\mathrm{C}$, which is not abundantly produced by PISNe. Their conclusion has since been adopted as positive evidence that VMSs dominated metal enrichment from the first stellar generation. On this assumption, Oh et al. (2001) argued that the $\mathrm{Fe}$ enrichment in the $\mathrm{P}$ inventory $([\mathrm{Fe} / \mathrm{H}]=-3)$ implies cumulative cosmic star formation activity sufficient to reionize the universe if all star formation is in VMSs. This result forms the empirical basis of the contention that VMSs could explain

\footnotetext{
${ }^{5}$ To avoid confusion with hydrogen, we adopt HF and LF to designate the $\mathrm{H}$ and $\mathrm{L}$ events posited by WQ00.
}

both the observed EMP metal abundances and reionization together (see points 3 and 5 in $\S 1$ ).

However, closer scrutiny shows that the $\mathrm{P}$ inventory does not match all the observations. The P inventory model is incompatible with the strong VMS hypothesis because VMSs have no significant post-He nuclear burning and therefore produce no $r$-elements (HW02). If VMSs produce all the $\mathrm{Fe}$ up to $[\mathrm{Fe} / \mathrm{H}] \sim-3$, the $r$-elements should be absent instead of appearing at $[r / \mathrm{Fe}] \gtrsim-0.5$ as observed. Progenitors with $M=8-40 M_{\odot}$ are needed in the first generation to provide the observed $r$-process elements (Truran et al. 2002), provided that the $r$-process mechanisms that operate at finite metallicity can be safely extended to $Z=0$. Thus, the strong VMS hypothesis is convincingly excluded, and the weak hypothesis is more naturally accommodated by the P inventory model.

The $\mathrm{P}$ inventory model also provides a means for testing the weak VMS hypothesis because it attributes virtually all the $\mathrm{Fe}$ to VMSs. To see why, we follow WQ00 and estimate the fraction of $\mathrm{Fe}$ in the $\mathrm{P}$ inventory that could be attributed to $\mathrm{HF}$ events. If we take the lowest $\epsilon(\mathrm{Ba})$ to represent the contribution of a single $\mathrm{HF}$ event, then 40 such events contribute to the scatter in $\epsilon(\mathrm{Ba})$ at $[\mathrm{Fe} / \mathrm{H}] \sim-3$ for a modest increase in $[\mathrm{Fe} / \mathrm{H}]$. This small gain in $\mathrm{Fe}$ enrichment limits the HF contribution to the $\mathrm{P}$ inventory to $\lesssim 10 \%$. Another approach to this proof comes from consideration of a model IMF. If we assume a Salpeter IMF with 1-260 $M_{\odot}$ and adopt HW02 yields for VMSs and a uniform Fe yield per star, $m_{\mathrm{Fe}}=0.07 M_{\odot}$ for $M=10-40 M_{\odot}$, we find that the VMS mass range produces $\sim \frac{2}{3}$ of the total Fe yield over time (more if the IMF slope or mass limits are changed to favor high masses). Thus, any element $\mathrm{X}$ with mass yield from VMSs similar to $\mathrm{Fe}$ should appear with the appropriate ratio $[\mathrm{X} / \mathrm{Fe}]$ in the $\mathrm{P}$ inventory.

In Figure 1 (top) we compare the PISN yields (HW02) to the EMP metal abundances from three sources (McWilliam et al. 1995; Carretta et al. 2002; Cayrel et al. 2004). We plot these samples separately and mark the theoretical PISN yields for three progenitor mass ranges with filled boxes. Yields for unobserved elements are included for completeness and marked with lighter colors. We see that the observed abundances are consistent across the different studies for elements that they have in common. Clearly, the high-mass PISNe match well with $\mathrm{Si}, \mathrm{Ca}$ (as pointed out by $\mathrm{Oh}$ et al. 2001), and to some extent with $\mathrm{Mn}$, but they are a poor match to the other elements. They overpredict $\mathrm{Cr}$, underpredict $\mathrm{V}$ and $\mathrm{Co}$, and produce little $\mathrm{Zn} .{ }^{6}$ The VMS models are a poor fit to the data, especially for the Fe-peak elements Co and $\mathrm{Zn}$. The separation by mass range emphasizes that enrichment by one or a few stars is a much poorer fit to the observed data than a massweighted (IMF-averaged) yield. Some elements are matched best by the lower mass range, while others favor the higher masses (which have Fe yields of up to $40 M_{\odot}$ ). Thus, the data appear to be inconsistent with a model, such as the P inventory, in which these metal-poor stars formed from gas that was enriched only once or a few times by VMS precursors. The generally poor match of PISNe yields leads us to conclude that VMSs were probably not the precursors of these EMPs.

The phenomenological model for $r$-process patterns by Fields et al. (2002) does not make specific reference to VMSs, but it is a good example of another class of models that can

\footnotetext{
${ }^{6}$ HW02 point out that PISN models may produce more $\mathrm{Zn}$ if neutrino-rich shocks are included in future models. The potential for some future model to better match the data does not presently constitute positive evidence of VMSs.
} 


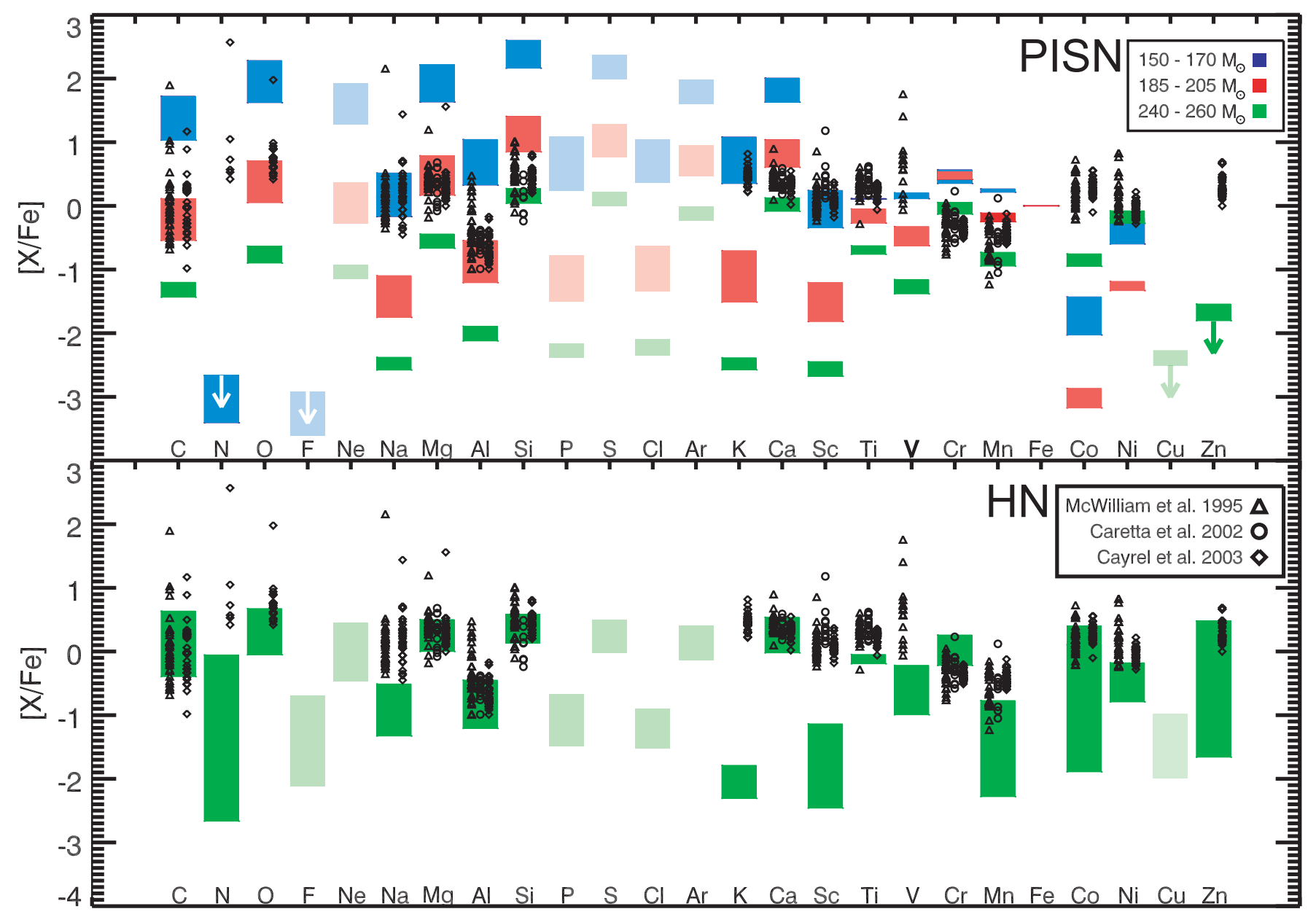

Fig. 1. - Observed element abundances for 63 metal-poor halo stars from McWilliam et al. (1995), Carretta et al. (2002), and Cayrel et al. (2004). These stars range over $[\mathrm{Fe} / \mathrm{H}]=-2$ to -4 . For duplicated stars, the higher resolution data from Cayrel et al. (2004) are used. Top: Comparison of the observed abundances with the theoretical yields for VMS pair-instability supernovae from $M=140$ to $260 M_{\odot}$. These yields are plotted separately for three ranges of stellar mass as indicated. Clearly, VMSs of a single mass cannot explain the observed yields. Also, the extreme odd-even effect predicted for VMSs is not apparent in the data. Bottom: Comparison of the observed abundances with the theoretical $\mathrm{HN}$ yields of $\mathrm{Cr}, \mathrm{Mn}, \mathrm{Co}$, and $\mathrm{Zn}$ from Umeda \& Nomoto (2003b), adjusted for small changes in $Y_{e}$, and for all other elements from Umeda \& Nomoto (2002), over the range $M=1-50 M_{\odot}$ and $E_{51}=1-100$. These yields provide a superior fit to the observed patterns, particularly for the Fe-peak elements $(\mathrm{Cr}-\mathrm{Zn})$.

test the VMS hypothesis. This study explains the $\sim 2$ dex scatter in $[r / \mathrm{Fe}]$ by a stochastic treatment of supernova enrichment that defines two types of $\mathrm{SN}$ with identical Fe mass yield but very different $[r / \mathrm{Fe}]$ (class A, high $[r / \mathrm{Fe}]$; class B, low or zero $[r / \mathrm{Fe}]$ ). Their artificial halo star sample reproduces the observed scatter in $[\mathrm{Eu} / \mathrm{Fe}]$ below $[\mathrm{Fe} / \mathrm{H}]=-2.5$ when A events comprise $\$ 5 \%$ of all $\mathrm{SNe}$. In this model, A events produce all the $r$-process elements, and B events make virtually all the $\mathrm{Fe}$. The assumed uniform $\mathrm{Fe}$ mass yield was adopted from SN 1987A (Woosley et al. 1994), but it also matches quite well to the Fe yields of VMS PISNe from 140 to $160 M_{\odot}$. The VMS PISNe are a good candidate for the Feproducing but $r$-process-poor B events, but in fact this model illustrates the difficulty with any model that attempts to attribute the EMP Fe budget to VMSs. As shown above (and in Fig. 1), the observed abundances of Fe-peak elements in EMPs are not a good match to PISNe. Although the absolute Fe yields from VMSs in the $140-160 M_{\odot}$ range serve well as $\mathrm{B}$ events, stars in this mass range produce negligible $\mathrm{Zn}$. The Fields et al. (2002) model is a generalization of the WQ00 P inventory model that accurately describes the observed scatter in $[r / \mathrm{Fe}]$ at low $[\mathrm{Fe} / \mathrm{H}]$. Both models thoroughly contradict the strong VMS hypothesis and are inconsistent with the weak version. The Fields et al. (2002) model is successful without including VMSs, so the general class of models it represents (including WQ00) does not provide positive evidence for VMSs.

Some of the recent detections of Galactic halo stars with $[\mathrm{Fe} / \mathrm{H}] \leq-3.0$ but enhanced $[\mathrm{C} / \mathrm{Fe}, \mathrm{N} / \mathrm{Fe}, \mathrm{O} / \mathrm{Fe}]$ present another puzzle to nucleosynthesis models. The lowest $[\mathrm{Fe} / \mathrm{H}]$ star (HE 0107-5240, $[\mathrm{Fe} / \mathrm{H}]=-5.3$; Christlieb et al. 2002) exhibits $[\mathrm{C} / \mathrm{Fe}]=4.0$ and $[\mathrm{N} / \mathrm{Fe}]=2.3(\mathrm{O}$ was not measured), so it may be more accurate to call this star "iron-poor" than "metal-poor." Recent high-resolution analyses of BPS CS 22949-037 (Norris et al. 2002; Depagne et al. 2002) and BPS CS 29498-043 (Aoki et al. 2002) show similar patterns. In their analysis of BPS CS 22949-037, Norris et al. (2002) point out a good fit of $[\mathrm{N} / \mathrm{Fe}]=2.6$ to the rotating zerometallicity VMS models of Fryer et al. (2001), in which primary $\mathrm{N}$ is produced by $\mathrm{C}$ and $\mathrm{O}$ mixing into the $\mathrm{H}$-rich shell. However, they note that this model overproduces $\mathrm{N}$ by a factor of 10 and that the star does not exhibit the exaggerated oddeven nuclei effect of PISNe. In a subsequent analysis of $[\mathrm{O} / \mathrm{Fe}]$ in this same star, Depagne et al. (2002) argue for a close match to an unpublished model of Heger \& Woosley with $M=35 M_{\odot}$. This model (Z35Z) matches the observed 
abundances of BPS CS 22949-037 when modified to include non-LTE effects for $\mathrm{Al}$ and $\mathrm{Na}$ and enhanced $\mathrm{N}$ production by $\mathrm{C}$ and $\mathrm{O}$ mixing into the $\mathrm{H}$-rich shell. Furthermore, this model produces $\mathrm{Zn}$ in the correct ratio to $\mathrm{Fe}$, while no PISN models can do so.

In a study of HE 0107-5240, Limongi et al. (2003) argue that this star cannot be fitted with the yields of a single supernova because the Fe-peak elements favor a deep mass cut (such that relatively more material is ejected), while the light elements favor a shallow one (to produce high $[\mathrm{C} / \mathrm{Fe}]$ ). Instead, they obtain a good fit for two SN sources: an earlier $15 M_{\odot}$ SN with a deep mass cut between the ejected material and the remnant that releases the heavier elements, and a later $35 M_{\odot} \mathrm{SN}$ with such a shallow mass cut that the supernova nearly fails to eject any metals at all. Although it requires two fine-tuned SN models to do so, their model matches HE 0107-5240 without recourse to VMSs. Umeda \& Nomoto (2003a) also find that the abundance pattern in this star fits nucleosynthesis from metal-free stars with $M=20-130 M_{\odot}$.

Because $r$-process enrichment is common in EMP stars and because PISNe experience no $r$-process (HW02), we can rule out the strong VMS hypothesis; clearly, $8-40 M_{\odot}$ stars must have existed in the generation that enriched the EMP stars. Furthermore, because the existing models with plausibly constructed IMFs in the weak VMS formulation must attribute all the early Fe to VMSe, they also fail to match the observed abundance patterns of EMP stars. Thus, we find that the nucleosynthesis constraints do not provide concrete, positive evidence for VMSs that cannot be explained by other means. Although ambiguities remain in the interpretation of the CNOrich EMPs, they clearly do not require VMSs and/or PISNe. Unless additional input physics in PISN models change the odd-even and Fe-peak characteristics of their yields, it appears the EMP stars do not support the VMS hypothesis.

\subsection{Hypernovae and Nucleosynthesis}

Umeda \& Nomoto $(2002,2003 \mathrm{a}, 2003 \mathrm{~b})$ and Nomoto et al. (2004) have developed an alternative explanation for EMP metal abundances. They argue that energetic supernovae, or hypernovae ( $\mathrm{HNe} ; E_{51}=10-100$, where $\left.E_{51}=E / 10^{51} \mathrm{ergs}\right)$ that appear to be connected with some low- $z$ gamma-ray bursts could be relevant for early nucleosynthesis if they exist at low metallicity. Empirical estimates based on light curves (Hamuy 2003) suggest that these events arise from supernovae with $E_{51}=10-30$ and progenitors of $M \gtrsim 15 M_{\odot}$. Since they have been found at low redshift, these objects are somewhat less speculative than VMSs and PISNe, which currently have only a theoretical motivation.

Umeda \& Nomoto (2003b) argue that the trends in the Fepeak elements in EMP stars reflect nucleosynthesis by $\mathrm{HNe}$ from zero-metallicity progenitors of $M<140 M_{\odot}$. In their models, the joint effects of a deeper mass cut between remnant and ejecta, mixing in the ejected material, and the fallback of some of the metals onto the remnant enhance the products of complete Si burning $(\mathrm{Zn}, \mathrm{Co})$ relative to the products of incomplete Si burning ( $\mathrm{Mn}, \mathrm{Cr}$ ). A higher explosion energy also increases the dilution mass (the mass of pristine gas into which the ejected metals are mixed). Thus, second-generation stars born of their products will possess lower $[\mathrm{Fe} / \mathrm{H}]$ than stars enriched by lower energy SN, with higher $[(\mathrm{Zn}, \mathrm{Co}) / \mathrm{Fe}]$ and lower $[(\mathrm{Cr}, \mathrm{Mn})]$ as $[\mathrm{Fe} / \mathrm{H}]$ decreases. This detail in the models may need to change in light of the tighter scatter and smoother trends in the EMP observations of Cayrel et al. (2004). However, in general, HNe appear to provide a superior match to the EMP data. Furthermore, Umeda \& Nomoto (2003b) argue that CNO-rich EMP stars can be explained with variations in explosion energy and the degrees of mixing and fallback in the ejected material.

For comparison with the PISN yields, Figure 1 (bottom) shows the HN models compared with the three EMP surveys. We display the $\mathrm{HN}$ yields for the Fe-peak elements $\mathrm{Cr}, \mathrm{Mn}$, $\mathrm{Co}$, and $\mathrm{Zn}$ resulting from explosions with $M=13-50 M_{\odot}$ and $E_{51}=1-100$ (UN03b). These elements are insensitive to the location of the mass cut in the supernova explosion, but they can vary with the electron mass fraction $\left(Y_{e}\right)$ in the ejected material, the effects of which we include in the plotted range to show the improved fit (e.g., Fig. 4 of Umeda \& Nomoto 2003b). The large explosion energies of hypernovae are needed to reproduce the observed $\mathrm{Zn}$. For all other elements, we plot the yields of Umeda \& Nomoto (2002), with $M=15-20 M_{\odot}, E_{51}=1-5$, and including mixing and fallback of the ejected material. While the $\mathrm{HN}$ phenomenon is gaining support from gamma-ray burst observations and theory, the HN explosion energy is still a free parameter in the Umeda \& Nomoto (2003b) models, and it is somewhat degenerate with stellar mass as a determining factor of the metal yields. There is also uncertainty associated with extrapolation from the low- $z$, roughly solar-metallicity conditions to $Z \leq 10^{-3} Z_{\odot}$. Although the apparent match of HNe to the EMP metal abundances is encouraging, they cannot yet be conclusively identified as the products of the first stars. Nevertheless, the possible existence of HNe with $M=10$ $50 M_{\odot}$ in the first generation is an important part of constructing a first-stars IMF without VMSs.

\subsection{Discussion of Nucleosynthesis Results}

We have reviewed the available evidence from stellar nucleosynthesis studies that bear on the mass function of the first stars. Oh et al. (2001) proposed that the correspondence between the HW02 PISN yields and the WQ00 prompt inventory implied that VMSs were the sources of $\mathrm{Fe}, \mathrm{Si}$, and $\mathrm{Ca}$ at $[\mathrm{Fe} / \mathrm{H}]<-3$. They used this $\mathrm{Fe}$ abundance to estimate the global ionizing photon budget available to reionize the universe. These conclusions are not supported by the broader sets of elemental abundances. In contrast, we draw the following conclusions from our examination of the nucleosynthetic evidence:

1. The common presence of $r$-process elements in halo EMPs (McWilliam et al. 1995; Burris et al. 2000) suggests progenitors of $8-40 M_{\odot}$, where the $r$-process elements are thought to form (Truran et al. 2002). This requirement is incompatible with the strong VMS hypothesis and emphasizes the importance of the lower mass limit of the IMF.

2. The relative abundances of Fe-peak elements in EMPs (especially Zn; Cayrel et al. 2004) imply Type II supernovae from massive stars, or energetic hypernovae with deep mass cuts and mixing in the ejected material (Umeda \& Nomoto 2003b). These abundances are not well fitted by the yields of pair-instability supernovae from VMSs. This is not a refutation of the weak VMS hypothesis because it does not constitute evidence that VMSs were not present at some level. It does imply that VMSs are not responsible for the bulk of the Fe at $[\mathrm{Fe} / \mathrm{H}]<-3$ (as suggested by WQ00 and Oh et al. 2001).

Much depends on the true nature of the Galactic halo EMP stars. If they do not, in fact, trace the enrichment of the first stellar generation, then we will be left with few, if any, such tracers and none that indicate VMSs. For example, damped 
Ly $\alpha$ systems (DLAs) in QSO spectra are often considered to be candidates for studies of primordial nucleosynthesis because they have low metallicity and, at high $z$, they may reflect early chemical enrichment. However, the existing literature on DLAs (Prochaska \& Wolfe 2002 and references therein) shows that they generally have $[\mathrm{Fe} / \mathrm{H}]>-2$, while the Galactic halo EMPs range down to -4 . Thus, they may not reflect the same primordial metal enrichment thought to be seen in the EMPs with $[\mathrm{Fe} / \mathrm{H}] \leq-3$. Furthermore, the selective depletion of $\mathrm{Fe}$ and other refractory elements onto dust grains in DLAs can change the Fe-peak abundance ratios in the gas and thus complicate comparisons with theoretical yields and other observations. Finally, direct ratios between $\mathrm{Zn}, \mathrm{Cr}$, and $\mathrm{Mn}$ in DLAs match the EMP stars that also have $[\mathrm{Fe} / \mathrm{H}]>$ -2 , above the metallicity where the qualitatively distinct behavior of EMPs appears. While Galactic stars and DLAs in this higher metallicity regime may derive their metals from similar sources and may in fact have unusual features, DLAs by themselves do not indicate that they have been enriched by PISNe. The presence of VMSs is also not required by either the observed metal ratios in QSO broad emission line regions (Venkatesan et al. 2004) or by [Si/C] ratios in high-redshift Lyman limit systems (Levshakov et al. 2003).

The recent studies (see $\S 2$ ) of reionization by VMSs have expanded on the reionization thesis offered by $\mathrm{Oh}$ et al. (2001), but they have relied for the most part on that initial study to ground their use of the VMS hypothesis in terms of the nucleosynthetic evidence. Although it has been evaluated by observers in their presentation of the data, the independent evidence for the VMS hypothesis has not been widely examined in the theoretical literature. Previous studies have in many cases met the WMAP and Gunn-Peterson constraints on the $\mathrm{H}$ I reionization history of the IGM, but they have done so by assuming extreme astrophysical parameters or by assuming the VMS hypothesis, which we find to have no firm external justification. We return to the issue of reionization by the first stars in $\S 5$.

\section{THE IMF OF THE FIRST STARS}

\subsection{Constructing an IMF}

With the nucleosynthetic results in mind, we can now construct a first-stars IMF that is consistent with the observations. To the observational constraints discussed in $\S 3.4$ we add the following theoretical motivations for constructing an IMF that is deficient in low-mass stars but not skewed to the VMS mass range:

3. The general astrophysical arguments (cooling, etc.) used to motivate a "top-heavy" IMF still apply. Apparently, forming massive stars at $Z=0$ is a fundamentally different process than it is today. While Tan \& McKee (2004) point out that formation feedback may limit the mass of metal-free stars to $\sim 30 M_{\odot}$, they also suggest that there is no known mechanism for halting the accretion below this range $\left(\sim 1-10 M_{\odot}\right)$ or fragmenting into smaller clumps. On general grounds, this justifies increasing the lower limit of the IMF.

4. As shown in Figure 2, the ionizing efficiency of metalfree stars increases rapidly from 1 to $50 M_{\odot}$, with a plateau at 100-120 $M_{\odot}$. Thus, we can construct an IMF that achieves an ionizing efficiency similar to even a "strong" VMS model without recourse to stars with $M \geq 140 M_{\odot}$.

We propose a first-stars IMF that embodies the observational constraints $(1,2)$ and the theoretical arguments $(3,4)$.

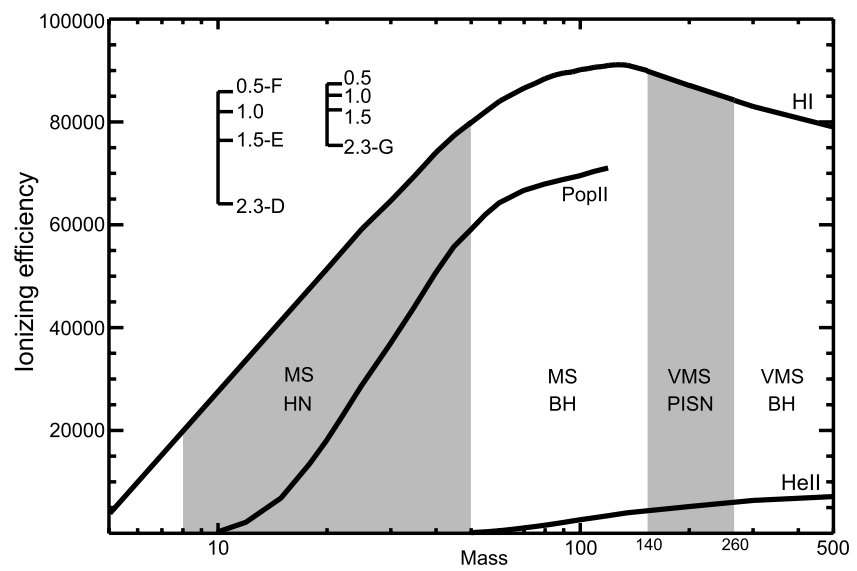

FIG. 2.-Number of $\mathrm{H}$ I and He II ionizing photons per baryon ("ionizing efficiency") as a function of stellar mass and integrated over the star's lifetime. Note the sharp rise from $M=10$ to $50 M_{\odot}$ and the gradual decline for $M>$ $120 M_{\odot}$, owing to asymptotic effective temperature gains and decreasing stellar lifetimes. Also shown at 10 and $20 M_{\odot}$ are the IMF-weighted values for IMF slope between 0.5 and 2.3 and the indicated lower mass limits (these are marked with their corresponding letters from Table 1 where applicable). The ionizing efficiency peaks before the VMS mass range and decreases steadily at higher mass. Thus, early reionization might be achieved by a "normal" IMF from which the low-mass stars are absent rather than a VMS IMF. We also show for comparison a characteristic curve for Population II, derived from models run with the Starburst99 code (Leitherer et al. 1999) and assuming standard mass loss and $Z=0.001$.

It is consistent with the available nucleosynthetic evidence, and it maximizes the ionizing photon efficiency to meet existing constraints on the reionization without violating those on IGM metal enrichment below $z \lesssim 6$ (Venkatesan \& Truran 2003). In Table 1 we present six new IMF cases that meet these criteria. There are four cases with Salpeter slope and varying upper and lower mass limits $(\mathrm{B}-\mathrm{D}, \mathrm{G})$ and two cases with varying slope $(\mathrm{E}-\mathrm{F})$ to capture the effects of proportionally more massive star formation in addition to variable mass limits. We also include a pure-VMS IMF (A) for comparison. Although it is tempting to change the functional form of the IMF from a power law to a more complicated function that favors high-mass stars, we avoid this for two reasons. First, the formation models have not given us specific reason to do so; they suggest that the mass limits could change but have not yet determined the shape of the distribution. Second, a more general function departing from a power law would introduce another parameter, and the IMF slope is already poorly constrained. Even if one knows the IMF slope or mass limits but not both, the inherent degeneracies in the problem may permanently preclude well-justified constraints on the other. A major part of the IMF $\left(M=50-140 M_{\odot}\right)$ leaves little or no nucleosynthetic trace, so the relative contributions of these stars to the ionizing radiation cannot be easily constrained. Thus, there remain significant uncertainties and ambiguities in constraining the first-stars IMF.

Figure 2 shows why our proposed IMF cases can achieve reionization results similar to VMSs. Here we show the lifetime-integrated ionizing photon production per baryon ("ionizing efficiency") in metal-free stars of 5-500 $M_{\odot}$. Up to $M=100 M_{\odot}$, the models are from Tumlinson et al. (2003); for $M>100 M_{\odot}$ they are from unpublished models based on the same evolution and stellar atmosphere code, checked for consistency against the results of Schaerer (2002). The notable features of this plot are the sharp rise to a peak at $M \simeq$ $120 M_{\odot}$, followed by a slow decline at higher mass. This effect 
TABLE 1

EFFects of Stellar IMFs on Reionization

\begin{tabular}{|c|c|c|c|c|c|c|c|c|c|}
\hline \multirow[b]{2}{*}{ Model } & \multirow{2}{*}{$\begin{array}{l}\text { IMF } \\
\left(M_{\odot}\right)\end{array}$} & \multirow[b]{2}{*}{$Q_{\mathrm{H} \mathrm{I}}$} & \multirow[b]{2}{*}{$Q_{\text {Не II }}$} & \multicolumn{3}{|c|}{$10^{7} \mathrm{yr}$ (INTRAhalo) } & \multicolumn{3}{|c|}{$10^{8} \mathrm{yr}($ HaLO-HaLO $)$} \\
\hline & & & & $z_{r, \mathrm{H}}$ & $z_{r, \mathrm{He}}$ & $\tau_{e}$ & $z_{r, \mathrm{H}}$ & $z_{r, \mathrm{He}}$ & $\tau_{e}$ \\
\hline А & $10^{2}-10^{3}$ & $1.6 \mathrm{E} 48$ & $3.8 \mathrm{E} 47$ & 13.4 & 10.3 & 0.134 & 15.4 & 13.8 & 0.157 \\
\hline В & $1-100$ & $1.1 \mathrm{E} 47$ & $1.5 \mathrm{E} 45$ & 11.4 & 0.5 & 0.095 & 11.8 & 1.6 & 0.102 \\
\hline С & $1-140$ & $1.5 \mathrm{E} 47$ & $3.3 \mathrm{E} 45$ & 11.5 & 0.9 & 0.097 & 12.1 & 3.9 & 0.109 \\
\hline D ...................... & $10-140$ & 4.3E47 & $9.8 \mathrm{E} 45$ & 12.0 & 1.6 & 0.105 & 13.7 & 7.0 & 0.128 \\
\hline 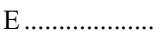 & $10-140, \alpha=1.5$ & $6.7 \mathrm{E} 47$ & $2.2 \mathrm{E} 46$ & 12.3 & 3.3 & 0.112 & 14.3 & 8.9 & 0.137 \\
\hline $\mathrm{F}$ & $10-140, \alpha=0.5$ & $8.9 \mathrm{E} 47$ & $3.8 \mathrm{E} 46$ & 12.6 & 4.4 & 0.117 & 14.7 & 10.0 & 0.143 \\
\hline 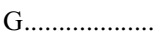 & $20-140$ & $6.1 \mathrm{E} 47$ & $1.5 \mathrm{E} 46$ & 12.3 & 2.5 & 0.110 & 14.2 & 8.0 & 0.134 \\
\hline
\end{tabular}

NoтE.-This table shows the IMFs considered in this work and the VMS IMF from Bromm et al. (2001), with the mass-normalized ionizing photon rates $Q_{i}$ (photons s ${ }^{-1} M_{\odot}^{-1}$ ) for $\mathrm{H} \mathrm{I}$ and $\mathrm{He}$ II at a starburst age of 1 Myr. The reionization redshifts, $z_{r}$, for $\mathrm{H}_{\mathrm{I}}$ and $\mathrm{He}$ II are also shown for each case, as well as the calculated electron-scattering optical depth $\tau_{e}$ (including the contribution from He II). We show results for two metalfree star formation cutoff times of $10^{7} \mathrm{yr}$ and $10^{8} \mathrm{yr}$, corresponding to the intrahalo and halo-halo metal enrichment timescales, respectively (see $\S 4.2$ ). All cases are for metal-free stars and an IMF given by $d N / d M \propto M^{-\alpha}$, with $\alpha=2.35$ unless otherwise stated.

is a consequence of the stellar lifetimes decreasing more rapidly than the main-sequence ionizing photon production increases. This curve shows that an IMF that excludes lowmass stars and their poor ionizing efficiency can closely approximate the total ionizing photon production of an IMF composed purely of VMSs.

Recently, Venkatesan \& Truran (2003) showed that VMSs may not be responsible for both reionization and IGM metal enrichment, since they produce only about 0.35 ionizing photons per baryon before they cease forming because of metal pollution (reionization at $z \sim 6$ is thought to require $\sim 10$ ionizing photons per baryon in the IGM, and more are required at higher redshifts). This contradiction would be resolved if the first stars were all VMSs with $M>260 M_{\odot}$ and reionization was accomplished by stars that emit ionizing radiation but release no metals. However, this way is probably closed because no mechanism has been proposed for forming $300 M_{\odot}$ stars without also forming $200 M_{\odot}$ stars in roughly equal or greater proportion. Furthermore, a primordial IMF composed exclusively of $M>260 M_{\odot}$ stars would never lead to the observed present-day star formation, as metals would not be released to trigger the transition from the first stars to a local IMF (the enrichment paradox; Schneider et al. 2002). The only way out of this paradox is to form stars below $M=$ $260 M_{\odot}$, where metals would be released by PISNe and influence later star formation. Although this argument could be modified by future results from formation models, this probably means that excluding $140-260 M_{\odot}$ also rules out more massive objects.

\subsection{The Duration of Metal-free Star Formation}

A key parameter in modeling the radiative and chemical input from the first stars is the duration of the metal-free phase of star formation. Given the complications of an unknown IMF, interstellar mixing, and halo merging history, this question is difficult to pose quantitatively. Here we make simplifying assumptions to estimate the enrichment time of small halos at $z=10-20$ to estimate the time during which metalfree gas is available to form stars.

As noted previously (Tumlinson et al. 2003; VTS03), the duration of the metal-free phase determines, with the IMF, the total output of ionizing photons from the first massive stars. As they die, these stars eject heavy elements from supernovae and enrich the surrounding gas within their own halo. These metals may also be transported across intergalactic space to enrich neighboring halos. Although these enrichment and transport processes are intrinsically complicated, we can make a few relevant calculations that constrain the duration of this metal-free epoch. Our calculations are similar in spirit to those of Scannapieco et al. (2003), but we make different assumptions.

First, we compute the global rate of metal enrichment per unit volume, based on an average star formation rate density (SFR), hereafter quoted in standard units of comoving $M_{\odot} \mathrm{yr}^{-1} \mathrm{Mpc}^{-3}$. By finding high-redshift galaxies through $B, V$, and $I$-band dropouts, Steidel et al. (1999) and Giavalisco et al. (2004) estimated an SFR $\approx 0.1$ in the range $3<z<6$. From the required rate of ionizing emission to reionize the IGM by $z=6$, Miralda-Escudé (2003) showed that the ionizing (comoving) emissivity cannot decline by more than a factor of 1.5 from $z=4$ up to $z=9$. Recent numerical simulations of galaxy formation (Gnedin 2000; Ricotti et al. 2002a, hereafter RGS; Ricotti et al. 2002b) allow us to extrapolate to the earliest epochs of galaxy formation $(z \approx 20-30)$, when star formation begins through $\mathrm{H}_{\mathrm{I}}$ and $\mathrm{H}_{2}$ cooling of protogalactic halos, with virial temperatures $T_{\mathrm{vir}}=10^{3}-10^{4} \mathrm{~K}$. RGS found that the average SFR increases monotonically, from $10^{-3}$ to $10^{-1}$ between $z=20$ and 10 , according to the approximate formula

$$
\mathrm{SFR} \simeq\left(0.003 M_{\odot} \mathrm{yr}^{-1} \mathrm{Mpc}^{-3}\right) 10^{(20-z) / 5}
$$

These simulations agree fairly well with the extended PressSchechter estimates of Scannapieco et al. (2003). To estimate the corresponding metal production, we adopt a fiducial SFR = $0.01 M_{\odot} \mathrm{yr}^{-1} \mathrm{Mpc}^{-3}$, with standard metal yield $y_{m}=0.00663$ for metal-free stars of $M=1-100 M_{\odot}$. After a time $t=$ $\left(10^{8} \mathrm{yr}\right) t_{8}$, the average IGM metallicity is

$$
\frac{Z_{\mathrm{IGM}}}{Z_{\odot}}=\frac{(\mathrm{SFR}) y_{m} t}{\Omega_{b} \rho_{\mathrm{cr}}(0.02)}=\left(5.4 \times 10^{-4}\right) \frac{\mathrm{SFR}}{0.01} t_{8} .
$$

The elapsed time between $z=20$ and 10 is $2.9 \times 10^{8} \mathrm{yr}$ for $H_{0}=70 h_{70} \mathrm{~km} \mathrm{~s}^{-1}, \Omega_{m}=0.3$, and $\Omega_{\Lambda}=0.7$. The above estimate shows that early star formation can raise the average 
IGM metallicity to values near the suggested critical threshold $Z_{\text {cr }} \approx 10^{-3.5} Z_{\odot}$ at which metal cooling may produce a transition to a different mode of star formation involving fragmentation into low-mass clumps (Omukai 2000; Bromm \& Loeb 2003; Schneider et al. 2002).

Complicating this estimate is the likelihood that primordial metal enrichment proceeded inhomogeneously (Scannapieco et al. 2002, 2003). Here we consider a halo with total mass $M_{h}=\left(10^{6} M_{\odot}\right) M_{6}$, in which $1.66 \times 10^{5} M_{6} M_{\odot}$ of baryons are distributed in $N$ clumps spread over a halo of virial radius $R_{\mathrm{vir}}$ and virial temperature $T_{\mathrm{vir}}$ :

$$
\begin{gathered}
R_{\mathrm{vir}}=\left(\frac{3 M_{h}}{72 \pi^{3} \rho_{m}}\right)^{1 / 3}=(165 \mathrm{pc}) M_{6}^{1 / 3}\left[\frac{20}{(1+z)}\right], \\
T_{\mathrm{vir}}=\left(\frac{G M_{h} m_{H}}{3 k_{B} R_{\mathrm{vir}}}\right)=(1060 \mathrm{~K}) M_{6}^{2 / 3}\left[\frac{(1+z)}{20}\right] .
\end{gathered}
$$

We assume cosmological densities $\rho_{m}=\left(1.88 \times 10^{-29} h^{2} \mathrm{~g}\right.$ $\left.\mathrm{cm}^{-3}\right) \Omega_{m}(1+z)^{3}$, with $\Omega_{m} h^{2}=0.135$ and $\Omega_{b} h^{2}=0.0224$ (for matter and baryons, respectively; Spergel et al. 2003) and a virialized density ratio $\rho\left(R_{\mathrm{vir}}\right) / \rho_{m}=18 \pi^{2}$. We set halo radius $R_{h}=R_{\text {vir }}$ and divide the matter into $N$ clumps, with contrast ratio $\rho_{\mathrm{cl}} / \rho_{h} \gg 1$, where $\rho_{h}=3 M_{h} / 4 \pi R_{h}^{3}$. We next assume that $5 \%$ of the halo's baryons undergo a burst of star formation at the center of the halo. As these stars eject their metals, a small fraction, $f_{\text {cap }}$, of these metals are captured by the nearest-neighbor clumps, while most are blown out into the IGM. The capture fraction may be estimated from the solid angle of the neighboring clump, of radius $r$, as seen from the halo center, $f_{\text {cap }} \approx\left(\pi r^{2} / d_{\mathrm{cl}}^{2}\right) / 4 \pi$. The mean distance between clumps within the halo is $d_{\mathrm{cl}} \approx\left(3 N / 4 \pi R_{h}\right)^{-1 / 3}$. We also have $N r^{3} \rho_{\text {cl }}=R_{h}^{3} \rho_{h}$. After some algebra, we find

$$
\begin{aligned}
f_{\text {cap }} & =\frac{1}{4}\left(\frac{3}{4 \pi}\right)^{2 / 3}\left(\frac{\rho_{h}}{\rho_{\mathrm{cl}}}\right)^{2 / 3} \\
& \approx 0.096\left(\frac{\rho_{h}}{\rho_{\mathrm{cl}}}\right)^{2 / 3} .
\end{aligned}
$$

We arrive at the simple result that the fraction of metals captured by the nearest-neighbor clump depends only on the density contrast fraction between the clump and halo.

For example, for the $10^{6} M_{\odot}$ halo, with total baryonic mass $M_{b}=1.66 \times 10^{5} M_{\odot}$, we assume $N=30$ clumps, each with $5530 M_{\odot}$ in baryons and a clump-interclump contrast ratio $\rho_{\mathrm{cl}} / \rho_{h} \approx 100$. If $5 \%$ of the halo's baryons $\left(8300 M_{\odot}\right)$ undergo a burst of star formation at the halo center, they will produce approximately $83 \mathrm{SNe}$ and $18.3 M_{\odot}$ of heavy elements $\left(y_{m} \approx\right.$ 0.00663 ). The nearest-neighbor clump will capture a fraction $f_{\text {cap }}=4.5 \times 10^{-3}$, for an "enriched" metallicity of $18.3(4.5 \times$ $\left.10^{-3}\right) / 5530 \approx 1.5 \times 10^{-5}$, or $7.4 \times 10^{-4} Z_{\odot}$. Thus, the subclumps within the halo will be enriched to $\sim 10^{-3}$ solar abundances on a timescale of a few Myr, the crossing time of the $\mathrm{SNe}$ ejecta across the halo.

A longer and perhaps more relevant timescale is that required to transport the heavy elements between halos, across the IGM. From recent simulations (Gnedin 2000; RGS), the comoving density of $10^{6} M_{\odot}$ star-forming halos at $z=15$ 20 is approximately $4 \pm 2 \mathrm{Mpc}^{-3}$, corresponding to a mean (physical) distance between halos of $d_{\text {halo }} \approx(30 \mathrm{kpc})[20 /$ $(1+z)]$. Thus, the ejected heavy elements, if they are transported at mean velocity $V_{f} \approx 100 \mathrm{~km} \mathrm{~s}^{-1}$, would not reach neighboring halos for over $10^{8} \mathrm{yr}$.
These neighboring halos may recede with the local Hubble flow, $H(z)=\left(3.43 \mathrm{~km} \mathrm{~s}^{-1} \mathrm{kpc}^{-1}\right) h_{70}$ at $z=20$. The Hubble expansion velocity between these halos could be as large as

$$
\begin{aligned}
V_{\mathrm{exp}} & \approx H_{0}(1+z)^{3 / 2} \Omega_{m}^{1 / 2} d_{\text {halo }} \\
& \approx\left(100 \mathrm{~km} \mathrm{~s}^{-1}\right)\left(\frac{1+z}{20}\right)^{1 / 2} .
\end{aligned}
$$

Alternatively, this region of space could have turned around from the expansion, depending on the extent and spatial correlations of the mass perturbations. Transporting the ejected metals across $30 \mathrm{kpc}$ at $z=20$ is not easy, given the amount of intervening intergalactic matter. A sphere of $1 \mathrm{kpc}$ (physical distance) surrounding a halo at $z=20$ contains $2 \times 10^{5} M_{\odot}$ of baryonic mass, at the mean hydrogen number density,

$$
n_{\mathrm{H}}=\left(1.52 \times 10^{-3} \mathrm{~cm}^{-3}\right)\left(\frac{1+z}{20}\right)^{3} .
$$

Any heavy elements ejected in SN explosions or outflows are accompanied by significant energy deposition. They rapidly sweep up many times their own mass, form cosmological blast waves, and eventually develop radiative shells (Ostriker \& Cowie 1981; Shull \& Silk 1981), which slow down as they expand.

Consider the $5 \%$ burst of star formation from the previous example. The resulting $83 \mathrm{SNe}$ would deposit an energy $E_{\mathrm{SN}} \approx\left(10^{54} \mathrm{ergs}\right) E_{54}$ into the IGM if we assume an energy of $10^{52}$ ergs per SN or HN (Umeda \& Nomoto 2002). An estimate of the blast wave extent comes from the noncosmological Sedov-Taylor formula,

$$
\begin{aligned}
R_{s} & =\left(\frac{2.02 E_{\mathrm{SN}} t^{2}}{\rho_{\mathrm{IGM}}}\right)^{1 / 5} \\
& \approx(7.35 \mathrm{kpc})\left(\frac{E_{54} t_{8}^{2}}{\delta}\right)^{1 / 5}\left(\frac{1+z}{20}\right)^{-3 / 5} .
\end{aligned}
$$

Here $\rho_{\mathrm{IGM}}=1.32 n_{\mathrm{H}} m_{\mathrm{H}}$ is the IGM mass density, $\delta$ is the overdensity relative to the mean, and $t=\left(10^{8} \mathrm{yr}\right) t_{8}$ is the time after the injection of SN energy. In reality, one must take cosmological expansion and IGM inhomogeneity into account, as well as the gradual injection of energy into the "superbubble" produced by multiple SNe over a total time of $\sim 5 \times 10^{7}$ yr.

From the above approximate formulae, we see that the expanding blast wave could take over $10^{8} \mathrm{yr}$ to contaminate neighboring halos. We conclude that the halo-to-halo enrichment timescale is likely to be on the order of $10^{8} \mathrm{yr}$. These simple calculations motivate our choices of $10^{7}$ and $10^{8} \mathrm{yr}$ as the duration of the first-stars epoch for primordial star formation. These timescales are in accord with those calculated from numerical simulations of halo self-enrichment by Wada \& Venkatesan (2003) and of interhalo enrichment by Bromm et al. (2003). The effects of these two timescales on the reionization models are discussed in $\S 5$.

In fact, our proposed first-stars IMF has another virtue along these lines. If most of the mass in the early IMF is formed into stars in the 50-140 $M_{\odot}$ mass range, the unique effects of a zero-metallicity IMF could persist longer than they would if the IMF favored VMSs. To see why, consider a case 
where all mass goes into forming stars with either $M=$ 50-140 $M_{\odot}$ or $M>260 M_{\odot}$. Since these stars collapse directly to black holes without releasing metals, the metal-free mode of star formation would be self-perpetuating. If this extreme IMF is modified to incorporate increasing but still relatively small numbers of SN II or HN progenitors, then metal enrichment will gradually achieve the global value of $Z=10^{-3.5} Z_{\odot}$ thought to curtail the unique low-metallicity star formation mechanisms. In a rough sense, this timescale is inversely proportional to the fraction of matter formed into stars that explode and release metals relative to those that do not. Thus, a significant advantage of the $M=30-140 M_{\odot}$ IMF is that the duration of metal-free star formation at early epochs could be longer because of the increased stellar lifetimes in this IMF relative to VMSs and because of the lower total metal yields.

\section{THE FIRST STARS AND REIONIZATION}

We have derived a first-stars IMF that is consistent with the available nucleosynthetic evidence, and we have estimated the time $\left(\sim 10^{7}-10^{8} \mathrm{yr}\right)$ over which metal-free stars can form in small primordial halos. We must now evaluate our proposed IMF in terms of reionization models to compare their effect on the IGM with that of VMSs. We hold fixed all the model assumptions except the IMF and the metal-enrichment timescale to determine the extent to which the IMF matters to the outcome.

We adopt the semianalytic stellar reionization model described in VTS03 for a concordance $\Lambda$ CDM cosmology. The growth of ionized regions is tracked by a Press-Schechter formalism in combination with numerical solutions for the growth of individual ionization fronts. For all cases here, the cosmological and astrophysical parameters are the same as in VTS03; we do this in order to consistently compare the effects of a VMS IMF with those proposed here (there is one exception, mentioned below). Specifically, our models are described by the parameter set $\left[\sigma_{8}, n, h, \Omega_{b}, \Omega_{\Lambda}, \Omega_{m}, c_{L}, f_{*}, f_{\mathrm{esc}}^{\mathrm{H}}, f_{\mathrm{esc}}^{\mathrm{He}}\right]=$ $[0.9,1.0,0.7,0.04,0.7,0.3,30,0.05,0.05,0.025]$, where $\sigma_{8}$ and $n$ are the usual normalization and index of the scalar matter power spectrum, $c_{L}$ is the space-averaged clumping factor of $\mathrm{H}$ II or He III (assumed to be the same here), $h$ is the Hubble constant in units of $100 \mathrm{~km} \mathrm{~s}^{-1} \mathrm{Mpc}^{-1}$, and $\Omega_{b}, \Omega_{\Lambda}$, and $\Omega_{m}$ represent the cosmological density parameters of baryons, the cosmological constant, and matter, respectively. The astrophysical parameters are $f_{*}$, the fraction of baryons forming stars in each halo, and $f_{\text {esc }}^{\mathrm{H}}$ and $f_{\text {esc }}^{\mathrm{He}}$, the escape fractions of $\mathrm{H} \mathrm{I}$ and He II ionizing photons from halos, respectively.

We allow star formation in all halos of virial temperature $\gtrsim 10^{3} \mathrm{~K}$ (rather than $10^{4} \mathrm{~K}$ as in VTS03), so as to have sufficient baryons in a $\sim 10^{6} M_{\odot}$ dark matter halo at $z=20$ for our adopted star formation efficiencies to form the most massive $\operatorname{star}\left(\sim 10^{3} M_{\odot}\right)$ in our IMF cases. Metal-free stars are assumed to form starting from $z \sim 20$ and last in each halo for either of two approximate timescales derived earlier for intrahalo and halo-halo enrichment, $10^{7} \mathrm{yr}$ and $10^{8} \mathrm{yr}$. Subsequent to this, the ionizing spectrum is switched to a representative example of Population II stars with metallicity $Z=0.001$ (Leitherer et al. 1999). Reionization is defined as the overlap of individual ionized regions of $\mathrm{H}$ II or $\mathrm{He}$ III as appropriate, when their volume filling factors equal unity (see VTS03).

Relative to the results of VTS03, reionization in our new models occurs earlier because of the lower halo mass threshold and the fixed metal-free star-forming phase in each halo before switching to a Population II ionizing spectrum. VTS03 assumed $T_{\text {vir }} \geq 10^{4} \mathrm{~K}$ and shut off metal-free star formation at fixed cosmological ages. Thus, in VTS03, the majority of small halos, in which most of the baryons reside until $z \lesssim 9$ when they merge into larger systems, did not experience any metal-free star formation, even if they had not undergone prior star formation or experienced pollution from neighboring halos. Our treatment here ensures that each halo experiences at least one episode of metal-free star formation prior to cosmological times of $\sim 1$ Gyr. The assumption of $T_{\mathrm{vir}} \geq 10^{3} \mathrm{~K}$ is somewhat more optimistic from the point of view of early reionization and relies on the assumption that halos with $T_{\text {vir }} \leq 10^{4} \mathrm{~K}$ can effectively cool with $\mathrm{H}_{2}$ (RGS). Other models (e.g., Haiman \& Holder 2003) have even more optimistically assumed star formation in halos down to $T_{\mathrm{vir}} \geq 10^{2} \mathrm{~K}$; these are not massive enough to form the most massive star in our IMF cases $\left(1000 M_{\odot}\right)$. The assumption that each new halo forms metal-free stars breaks down at $z \simeq 9$, when the earliest small halos, presumably metal-enriched from their first star formation, begin to coalesce into larger objects, which are presumably never metal-free. Our models reionize early enough to avoid this complication (see below).

In Table 1, we display the various IMFs considered in this work. We include newly calculated cases that are motivated by the nucleosynthetic data ( $(4.1)$ and cases that represent a topheavy IMF, in the form either of a higher stellar mass range or of a flatter IMF slope, $\alpha$. Also shown are the ionizing photon rates, $Q_{i}$, for $\mathrm{H}$ I and $\mathrm{He}$ II from synthetic stellar clusters at a starburst age of $1 \mathrm{Myr}$ in units of photons s ${ }^{-1} M_{\odot}^{-1}$. We also show the $\mathrm{H}$ I and $\mathrm{He}$ II reionization redshifts, $z_{r}$, for each case and the total associated $\tau_{e}$ (including the $\sim 10 \%-15 \%$ contribution from $\mathrm{He}$ II), assuming that the post-recombination IGM ionization fraction is about $10^{-4}$ over $z \sim 20-1000$. The equivalent ionizing rates for Population II are $Q_{\mathrm{HI}} \sim 7.8 \times$ $10^{46} \mathrm{~s}^{-1} M_{\odot}^{-1}$ and $Q_{\text {He II }} \sim 1.3 \times 10^{40} \mathrm{~s}^{-1} M_{\odot}^{-1}$ after the transition from Population III to Population II. ${ }^{7}$ We see that an IMF derived from the EMP nucleosynthetic constraints and lacking in low-mass stars can generate sufficiently early reionization and values of $\tau_{e} \geq 0.10$. This is consistent with current data on reionization without resorting to a top-heavy IMF; high ionizing efficiency need not arise from VMSs only. We also note that the uncertainty on $\tau_{e}$ from WMAP arises from a broad probability distribution and that it is degenerate with $n_{s}$. Recently, $\tau_{e}=0.12_{-0.06}^{+0.08}$ has been determined for WMAP plus the three-dimensional matter power spectrum from SDSS (Tegmark et al. 2003). Our models reproduce the WMAP result at the $1 \sigma$ confidence level and match the Tegmark et al. (2003) value without resort to VMSs. In fact, the close agreement achieved by our IMF suggests that the astrophysical parameters (star formation efficiency and escape fraction) are now the limiting factors in achieving good constraints on reionization. We also note that the models from VTS03 produced $\tau_{e} \sim 0.08$ with a more restrictive halo virial temperature cutoff $\left(10^{4} \mathrm{~K}\right)$ and a Salpeter IMF. The difference between this $\tau_{e}$ and model B shows that the more optimistic halo prescription of the present study contributes only a modest amount.

An important caveat for the results here is that the IGM, once reionized completely in $\mathrm{H}$ or $\mathrm{He}$, stays ionized. For $\mathrm{H} \mathrm{I}$, if reionization occurs once in the model at some redshift, e.g., $z=15$, and if metal-free stars are allowed to keep

\footnotetext{
${ }^{7}$ For comparison, the reionization models presented by VTS03 produced $z_{r, \mathrm{H}} \simeq 7$ assuming a Salpeter IMF with $1-100 M_{\odot}$, halos with $T_{\mathrm{vir}}>10^{4} \mathrm{~K}$, and a Population III to Population II transition time of $10^{8} \mathrm{yr}$.
} 
forming subsequently for $10^{7}-10^{8} \mathrm{yr}$ in each new halo, the model IGM does not recombine and stays ionized, even though recombinations are explicitly allowed. This result is probably valid for $\mathrm{H}_{\mathrm{I}}$ in the underdense voids, which represent most of the volume in the IGM. In the case of $\mathrm{H}$ I in dense regions and $\mathrm{He}$ II in general, this behavior may be suspect because of high recombination rates at typical IGM densities at $z \gtrsim 5$ (Shull et al. 2004). However, even if He III partially recombined in those of our cases in which He II reionization occurs early, the contribution to $\tau_{e}$ would be affected at less than the $10 \%$ level. Therefore, our numbers for He II should be considered only for the purposes of comparison between various IMFs. This is especially true at $z \lesssim 6$, where we have not included the ionizing radiation from QSOs. The simple reionization history in our models also does not reproduce the known $\mathrm{H}$ I Gunn-Peterson opacity at $z \sim 6$ or the observed IGM temperatures at $z=2-4$. These constraints would require additional input into the models, such as the contribution of quasars to the ionizing photon budget, or a more complicated reionization history than we explore here. Models that attempt to match all available constraints exist in the literature (see $\S 2$ ). The additional complications and new parameters would, if they were included in our model, needlessly distract from the goal of directly comparing the different IMF cases with the same minimal set of cosmological and astrophysical parameters. We also note that the $z \simeq 6$ Gunn-Peterson constraint can be met by a modest amount of recombination at $z=7-9$, perhaps coincident with the expected Population III to Population II transition, with only a modest effect on $\tau_{e}$. This is possible because even a trace neutral fraction is sufficient to provide $\tau_{\mathrm{GP}} \sim 1$ with negligible effect on the ionized fraction that scatters photons from the CMB. Because these external constraints can be met with minor adjustments to our model and because they are incidental to the IMF comparisons, we do not include them here.

\section{CONCLUSIONS AND DISCUSSION}

We close by drawing some important conclusions from the foregoing results and discussion. These are:

1. We should be distinguish two versions of the VMS hypothesis: the "strong" version ("the first stars were exclusively VMSs") and the "weak" version ("the first stars included VMSs and stars with $M<140 M_{\odot}$ "). This distinction is necessary because the radiative and nucleosynthesis properties of metal-free stars vary widely across the mass function. We have demonstrated that these two versions have different testable predictions and can be independently falsified.

2. The existing samples of metal abundances in EMP Galactic halo stars, which are commonly thought to trace the enrichment products of the first generation of stars, are better matched to the products of Type II SNe and hypernovae like those seen at low redshift. Specifically, the Fe-peak and $r$-process abundances in EMPs are not well matched by VMSs.

3. Because they are efficient sources of ionizing photons, VMSs have been proposed as the ionizing sources for the first stage of the IGM reionization and the only means of generating large electron-scattering optical depths seen in CMB maps. By excluding the low-mass end of the IMF, as suggested by models of primordial protostars, we have constructed IMF cases that are just as efficient as VMSs at converting baryons to ionizing photons. Thus, constraining the lower mass limit of the IMF is perhaps more important for reionization than a constraint on the upper limit.
4. Direct comparisons of our test IMFs in semianalytic reionization models show that without VMSs these models can reproduce $\tau_{e}$ in the observed range, and similar to VMS models with the same astrophysical parameters. We can simultaneously reproduce the nucleosynthesis and reionization constraints with a single IMF. We confirm earlier studies (VTS03; Wyithe \& Loeb 2003), which found that the lower end of the WMAP range of $\tau_{e}$ can be reproduced without recourse to a nonstandard IMF.

5. With simple metal transport calculations, we derive a timescale for metal-free star formation of $\sim 10^{7}-10^{8} \mathrm{yr}$. By effectively decoupling the earliest stages of ionization and metal enrichment, our proposed IMF can allow the period of metal-free star formation to persist longer than an IMF composed of VMSs, and perhaps long enough to fully reionize the IGM before self-enrichment of halos permanently curtails metal-free star formation.

We have shown that the nucleosynthesis constraints exclude the strong VMS hypothesis and have difficulty accommodating the weak VMS hypothesis. We have also shown that an IMF without VMSs can reproduce $\tau_{e} \sim 0.08$ if it includes stars with $M \lesssim 10 M_{\odot}$ and $\tau_{e} \sim 0.10-0.14$ if it does not. Rather than provide strong evidence for the VMS hypothesis, the existing $\tau_{e}$ values instead argue against low-mass stars but provide little constraint on massive stars in the IMF. In light of the metal-free star formation models, both the high- and lowmass ends of the stellar mass function are poorly constrained. It is only when the nucleosynthetic information is added that we can say anything about the IMF.

With these uncertainties in mind, we consider how future developments might bear on the first-stars IMF. First, a tighter range of $\tau_{e}$ is necessary to constrain the ionizing efficiency of the first stars and, indirectly, the IMF. Such an improvement might come with additional WMAP data, including polarization correlations. However, the greatest need is for consistent results from the various combinations of the $\mathrm{CMB}$ and additional data (i.e., matter power spectrum, $\operatorname{Ly} \alpha$ forest) that can constrain $\tau_{e}$. Uncertainty of $\sigma_{\tau} \lesssim 0.02$ on $\tau_{e}$ would leave the astrophysical parameters in a reionization model as the limiting uncertainties (Venkatesan 2002). It is important to point out that a low $\tau_{e}$ does not, by itself, disfavor the VMS hypothesis. Because their ionizing efficiency is lower than the peak (see Fig. 2), a strong VMS model could give a low $\tau_{e}$ with appropriately low star formation efficiency or photon escape fraction (if reionization is late), or with a short time available for metal-free star formation. Unless it settles on an extreme high value, $\tau_{e}$ is not likely by itself to provide decisive constraints on the IMF.

This latter point emphasizes the critical importance of robust constraints from nucleosynthesis as the remaining means of discriminating degenerate IMF cases (such as a strong VMS model vs. a weak VMS model without $M<10 M_{\odot}$; see Table 1). At the present time, EMP stars appear to exclude VMSs. This could change because of developments in the observations (new EMP stars with lower $[\mathrm{Fe} / \mathrm{H}]$ ) or refinements in the theory (reconciliation of PISNe to the Fe-peak elements would revitalize the weak VMS hypothesis). A particular concern would be evidence that the sample of EMP stars we presently take as the "second generation" is not, in fact, the product of the first stars. Beyond their chemically primitive atmospheres and ages, there is no hard evidence for this assumption. A new sample of metal-poor stars with $[\mathrm{Fe} / \mathrm{H}]<-4$ could change the outcome if they were better 
matched to the yields of PISNe and/or clearly devoid of $r$-process elements. There is also room for significant progress on the theoretical front: additional physics such as asymmetry, mass loss, and rotation could be added to the hypernova and PISN models, perhaps with a corresponding change in the logical basis of the VMS hypothesis.

The reionization results in Table 1 and the ionizing efficiencies in Figure 2 suggest another observational discriminant between our proposed first-stars IMF and one composed purely of VMSs. Although the $\mathrm{H}$ I ionizing efficiency of VMSs declines from the peak at $M \sim 120 M_{\odot}$, the ionizing efficiency for He II continues to increase into the VMS range. This feature of VMSs has two potential observational consequences. First, VMSs will be more efficient at producing the He II $1640 \AA$ recombination line, which has been suggested as a key direct signature of the first stars (Tumlinson et al. 2001; Schaerer 2002). Although the many degeneracies in the age and metallicity dependence of the $\lambda 1640$ line luminosity could give an ambiguous result, an unusually high value could be uniquely attributed to VMSs. The second possible signature is more speculative: the $\mathrm{He}$ II Gunn-Peterson test at high redshift is, in principle, sensitive to the spectrum of the first ionizing sources. A VMS IMF may reionize He II in the IGM before $z=6$, while a non-VMS IMF may or may not (see Table 1). Even if both IMFs can produce early He II reionization, it is still possible to discriminate between them using a GunnPeterson test. To see why, we recall the ratio $\eta=N_{\mathrm{He} \mathrm{II}} / N_{\mathrm{H} \text { I }}$ defined by Fardal et al. (1998) to characterize the ionizing sources of the IGM. Harder QSO-type spectra have $\eta \simeq$ $10-30$, while ordinary stars produce $\eta \simeq 100-400$. The increased He II ionizing efficiency of metal-free stars in a normal IMF can produce $\eta \sim 30$ (Tumlinson \& Shull 2000). By excluding inefficient low-mass stars, our proposed first-stars IMF gives $\eta \sim 20$. A pure VMS IMF could result in $\eta \lesssim 5$. Recent work at $z \sim 3$ with the Far Ultraviolet Spectroscopic Explorer (Shull et al. 2004; Zheng et al. 2004) has shown that $\eta<10$ and $\eta>30$ can be distinguished. The feasibility of this test would depend largely on the chance combination of a QSO with $z=6-8$ and little intervening absorption of the near-ultraviolet continuum by the common Ly $\alpha$ forest or Lyman limit systems at $z=1-2.5$. Although such a coincidence is unlikely, QSOs discovered at $z=6-8$ should be observed in the near-UV to qualify them for this test. Obtaining adequate $\mathrm{S} / \mathrm{N}$ to accurately measure the predicted low values of the $\mathrm{He}$ II opacity would likely require a large collecting area in the space ultraviolet, perhaps from a successor to the Hubble Space Telescope. If the practical problems can be overcome, the direct detection of He II $\lambda 1640$ and the high- $z$ He II Gunn-Peterson test provide some means of testing the first-stars IMF.

Finally, we add that, as a theoretical point of interest, the lower mass limit of the IMF can be just as important as the better studied upper limit because it can determine the total ionizing efficiency of the population. The lowest mass achievable by metal-free stars may depend on the details of primordial cooling, rotation, fragmentation, feedback, and magnetic fields in unknown ways. Attention has been focused on modeling the formation of very massive first stars; our results indicate that potential mechanisms for forming low-mass metal-free stars should be pursued. This is especially true if $\tau_{e}$ settles to a high value that requires high ionizing efficiency and the present nucleosynthesis constraints on the strong VMS hypothesis are confirmed.

Our proposed IMF can solve some key puzzles in the study of the first stars and reionization. However, there are still significant open questions about the formation of metal-free stars (especially the feedback mechanisms that influence their final masses) and the unknown time over which metal-free star formation persists. Furthermore, there may be no high-redshift direct tracers that can independently constrain the proposed IMF, especially since its most salient feature is the absence of stars with $1-10 M_{\odot}$ that are not directly observable at high redshift. Until the launch of the James Webb Space Telescope, which may be able to directly detect the first stars, future work should focus on more sophisticated treatments of the indirect tracers, particularly the EMP stars, as sensitive indicators of the first stellar generation. Nonetheless, we have shown that VMSs are not needed for reionization if the IMF is suitably constructed and that such an IMF is better justified on the grounds of the nucleosynthetic data than either version of the VMS hypothesis. In the future, better understanding of primordial star formation, more refined models of PISNe, or a new regime of extremely metal-poor stars with more VMSlike metal abundances may change the picture. For now, we conclude only that our proposed IMF is better matched to the available constraints.

We acknowledge helpful discussions with Jim Truran and constructive comments from Sergei Levshakov, Avi Loeb, and an anonymous referee. A. V. is supported by an NSF Astronomy and Astrophysics Postdoctoral Fellowship under award AST 02-01670. J. M. S. acknowledges support at the Colorado astrophysical theory program from NASA LTSA grant NAG5-7262 and NSF grant AST 02-06042.

\section{REFERENCES}

Abel, T., Bryan, G., \& Norman, M. L. 2002, Science, 295, 93

Aoki, W., Norris, J. E., Ryan, S. G., Beers, T. C., \& Ando, H. 2002, ApJ, 576, L141

Becker, R., et al. 2001, AJ, 122, 2850

Bromm, V., Kudritzki, R.-P., \& Loeb, A. 2001, ApJ, 552, 464

Bromm, V., \& Loeb, A. 2003, Nature, 425, 812 2004, NewA, 9, 353

Bromm, V., Yoshida, N., \& Hernquist, L. 2003, ApJ, 596, L135

Burris, D., Pilachowski, C. A., Armandroff, T. E., Sneden, C., Cowan, J. J., \&

Roe, H. 2000, ApJ, 544, 302

Carretta, E., Gratton, R., Cohen, J. G., Beers, T. C., \& Christlieb, N. 2002, AJ, 124,481

Cayrel, R., et al. 2004, A\&A, 416, 1117

Cen, R. 2003a, ApJ, 591, 12

. 2003b, ApJ, 591, L5

Christlieb, N., et al. 2002, Nature, 419, 904

Ciardi, B., Ferrara, A., \& White, S. D. M. 2003, MNRAS, 344, L7
Depagne, E., et al. 2002, A\&A, 390, 187

Djorgovski, S. G., Castro, S. M., Stern, D., \& Mahabal, A. 2001, ApJ, 560, L5 Fardal, M., Giroux, M. L., \& Shull, J. M. 1998, AJ, 115, 2206

Fields, B. D., Truran, J. W., \& Cowan, J. J. 2002, ApJ, 575, 845

Fryer, C. L., Woosley, S. E., \& Heger, A. 2001, ApJ, 550, 372

Fukugita, M., \& Kawasaki, M. 2003, MNRAS, 343, L25

Giavalisco, M., et al. 2004, ApJ, 600, L103

Gnedin, N. Y. 2000, ApJ, 535, 530

Gnedin, N. Y., \& Ostriker, J. P. 1997, ApJ, 486, 581

Haiman, Z., \& Holder, G. P. 2003, ApJ, 595, 1

Hamuy, M. 2003, ApJ, 582, 905

Heger, A., \& Woosley, S. E. 2002, ApJ, 567, 532 (HW02)

Hui, L., \& Haiman, Z. 2003, ApJ, 596, 9

Kaplinghat, M., Chu, M., Haiman, Z., Holder, G. P., Knox, L., \& Skordis, C. 2003, ApJ, 583, 24

Kogut, A., et al. 2003, ApJS, 148, 161 
Leitherer, C., et al. 1999, ApJS, 123, 3

Levshakov, S., Agafonova, I. I., Centurión, M., \& Molaro, P. 2003, A\&A, 397,851

Limongi, M., Chieffi, A., \& Bonifacio, P. 2003, ApJ, 594, L123

Marigo, P., Girardi, L., Chiosi, C., \& Wood, P. R. 2001, A\&A, 371, 152

McWilliam, A. 1997, ARA\&A, 35, 503

McWilliam, A., Preston, G. W., Sneden, C., \& Searle, L. 1995, AJ, 109, 2757

Miralda-Escudé, J. 2003, ApJ, 597, 66

Nomoto, K., Maeda, K., Umeda, H., Tominaga, N., Ohkubo, T., Deng, J., \& Mazzali, P. A. 2004, in Carnegie Obs. Astrophys. Ser. 4, Origin and Evolution of the Elements, ed. A. McWilliam \& M. Rauch (Pasadena: Carnegie Obs.), 1, http://www.ociw.edu/ociw/symposia/series/symposium4/proceedings.html

Norris, J. E., Ryan, S. G., Beers, T. C., Aoki, W., \& Ando, H. 2002, ApJ, 569, L107

Oh, S. P., Nollett, K. M., Madau, P., \& Wasserburg, G. J. 2001, ApJ, 562, L1

Omukai, K. 2000, ApJ, 534, 809

Omukai, K., \& Palla, F. 2003, ApJ, 589, 677

Ostriker, J. P., \& Cowie, L. L. 1981, ApJ, 243, L127

Prochaska, J. X., \& Wolfe, A. M. 2002, ApJ, 566, 68

Qian, Y.-Z., \& Wasserburg, G. J. 2001, ApJ, 559, 925 2002, ApJ, 567, 515

Ricotti, M., Gnedin, N. Y., \& Shull, J. M. 2002a, ApJ, 575, 33 (RGS) 2002b, ApJ, 575, 49

Scannapieco, E., Ferrara, A., \& Madau, P. 2002, ApJ, 574, 590

Scannapieco, E., Schneider, R., \& Ferrara, A. 2003, ApJ, 589, 35

Schaerer, D. 2002, A\&A, 382, 28

Schneider, R., Ferrara, A., Natarajan, P., \& Omukai, K. 2002, ApJ, 571, 30

Shull, J. M., \& Silk, J. 1981, ApJ, 249, 26
Shull, J. M., Tumlinson, J., Giroux, M. L., Kriss, G. A., \& Reimers, D. 2004, ApJ, 600, 570

Somerville, R. S., Bullock, J. S., \& Livio, M. 2003, ApJ, 593, 616

Spergel, D., et al. 2003, ApJS, 148, 175

Steidel, C. C., Adelberger, K. L., Giavalisco, M., Dickinson, M., \& Pettini, M. 1999, ApJ, 519, 1

Tan, J. C., \& McKee, C. F. 2002, in AIP Conf. Proc. 666, The Emergence of Cosmic Structure, ed. S. S. Holt \& C. S. Reynolds (Melville: AIP), 93 2004, ApJ, 603, 383

Tegmark, M., et al. 2004, Phys. Rev. D, 69, 103501

Truran, J. W., Cowan, J. J., Pilachowski, C. A., \& Sneden, C. 2002, PASP, 114,1293

Tumlinson, J., Giroux, M. L., \& Shull, J. M. 2001, ApJ, 550, L1

Tumlinson, J., \& Shull, J. M. 2000, ApJ, 528, L65

Tumlinson, J., Shull, J. M., \& Venkatesan, A. 2003, ApJ, 584, 608 (TSV03)

Umeda, H., \& Nomoto, K. 2002, ApJ, 565, 385 2003a, Nature, 422, 871

2003b, ApJ, submitted (astro-ph/0308029) (UN03b)

Venkatesan, A. 2002, ApJ, 572, 15

Venkatesan, A., Schneider, R., \& Ferrara, A. 2004, MNRAS, 349, L43

Venkatesan, A., \& Truran, J. W. 2003, ApJ, 594, L1

Venkatesan, A., Tumlinson, J., \& Shull, J. M. 2003, ApJ, 584, 621 (VTS03)

Wada, K., \& Venkatesan, A. 2003, ApJ, 591, 38

Wasserburg, G. J., \& Qian, Y.-Z. 2000, ApJ, 529, L21 (WQ00)

Woosley, S. E., Wilson, J. R., Mathews, G. J., Hoffman, R. D., \& Meyer, B. S. 1994, ApJ, 433, 229

Wyithe, S., \& Loeb, A. 2003, ApJ, 586, 693

Zheng, W., et al. 2004, ApJ, 605, 631 